\title{
Folate Deficiency and Prevalence of Neural Tube Defects [NTDs] in Georgia
}

Robizon Tsiklauri*, P. Imnadze, L. Jijeishvili, J. Morgan. N. Saganelidze

NCDC Georgia; USA CDC; LTD "Vistamedi" lab. Company

\section{Abstract}

Background: Until 2015, systematic statistical data on micronutrient deficiency was not available in Georgia, to provide developing national strategy. In the same year, the National Centre for Disease Control and Public Health of Georgia (NCDC) in collaboration with the USA CDC launched the project "Strengthening surveillance of micronutrient deficiency in Georgia".

Methodology: Sentinel surveillance approach was used by selecting 8 sentinel sites from 4 regions, 2 sites in each region ( 1 pediatric and 1 antenatal clinic). Folate and iron deficiencies were studied in pregnant women ( $1^{\text {st }}$ trimester) and iron deficiency was measured in children (12-23 months old). Ferritin concentration in plasma with cut-off points of $<12 \mu \mathrm{g} / \mathrm{l}$ was used for Iron deficiency measure in toddlers, and $<15 \mu \mathrm{g} / \mathrm{l}$ in pregnant. $<3.0 \mathrm{ng} / \mathrm{ml}$ was considered as a cut-off point of Folate deficiency in pregnant. For anemia detection for the both target groups (children U2; pregnant) has been used Hb cut-off points of $<11 \mathrm{~g} / \mathrm{l}$.Hemoglobin was tested using HemoQue and Multi-analyzer techniques at the sentinel sites.

Findings: In 2016-2017, Blood hemoglobin was measured among 939 children 12 to 23 months of age. $34 \%$ were anemic. Furthermore, 478 were tested for iron deficiency and $84 \%$ of them were identified as iron deficient. Hemoglobin was tested among 2,790 pregnant women and $7.4 \%$ of them were found anemic. Additionally, 483 of 2,790 pregnant women were tested for iron and folate deficiencies. $61 \%$ were iron deficient, and $26.4 \%$ tested positive for folate deficiency. Neural tube defects (NTDs) prevalence per 1000 live births registered in sentinel sites was high 2.7 .

Conclusion: Our preliminary results show that anemia and iron deficiency are prevalent among both pregnant women and children of the specified age group in Georgia. Additionally, folate deficiency was quite common during the1st trimester of pregnancy. Our findings will inform public health policy decision makers to take relevant decisions on required interventions, such as health education, distribution of relevant supplements, and food fortification.

\section{Introduction}

Globally, micronutrient deficiencies affects approximately 2 billion people. Major morbidity and mortality are associated with vulnerable populations notably children under five and pregnant woman. Major micronutrient deficiencies include iron, folate and iodine [1]. In 2013 , iron deficiency anemia affects $27 \%$ of the world's population. More than $89 \%$ of the burden comprises low income countries. Irondeficiency anemia causes more than $60 \%$ of anemia in the general population. Children under five and women of reproductive age are particularly vulnerable by anemia, which occurs when red blood cells are below the normal level.

Epidemiological studies for assessing the micronutrient status among the population in the South Caucasus region which includes Georgia, Azerbaijan, and Armenia, are limited. Reports from Armenia (2000) estimated the prevalence of anemia to be $12 \%$ and $12.4 \%$ among pregnant women and non-pregnant women, respectively and to be $23.9 \%$ in children under five [2].

A nationwide survey in Georgia reported prevalences of $22.8 \%$ anemic children, $25.6 \%$ in pregnant and $36.6 \%$ in non-pregnant women [3].

Iron is an essential element for the biosynthesis of blood hemoglobin The symptoms of iron deficiency anemia can be mild at first and are not diagnosed until they have a routine blood test. Potential health complications of iron deficiency anemia include rapid or irregular heartbeat, pregnancy complications of premature birth or low birth weight, and delayed growth in infants and children [4].

Folate insufficiency manifests in neural tube defects (NTDs), which is caused by low concentration of vitamin B9 (folate) in blood.
Approximately 190,000 neonates are born with NTD in low Income Countries. TDs are serious and most common consequence of folic acid deficiencies. NTDs occur when neural tube closure is completed by embryonic day 28 of pregnancy and arise when the neural tube cannot close properly. The most common NTDs are the following: anencephaly and spina bifida [5].

\section{Potential Target Groups}

Potential target groups for surveillance of micronutrient deficiency are infants, toddlers, preschool children, school-age children, and women of childbearing age (ref). Toddlers are vulnerable to micronutrient deficiencies, are accessible for the assessment in child health clinics and community surveys, and are an indicator of risk in the general population. Surveillance of iron deficiency is focused on these vulnerable groups. In the present study women at the 1st trimester of pregnancy were studied.

\section{Gap in knowledge}

The last assessments of nutritional status have been done in Georgia in 2009, but they do not contribute substantially to the estimation of nationwide prevalence rates of nutritional outcomes.

"Corresponding Author: Dr. Robizon Tsiklauri, NCDC Georgia; USA CDC; LTD "Vistamedi" lab. Company; E-mail: robizont@yahoo.com

Citation: Tsiklauri R, Imnadze P, Jijeishvili L, Saganelidze JM (2019) Folate Deficiency and Prevalence of Neural Tube Defects [NTDs] in Georgia. Int J Clin Nutr Diet 5: 143. doi: https://doi.org/10.15344/2456-8171/2019/143

Copyright: (C) 2019 Tsiklauri et al. This is an open-access article distributed under the terms of the Creative Commons Attribution License, which permits unrestricted use, distribution, and reproduction in any medium, provided the original author and source are credited. 
Page of

Currently we are establishing national nutrition monitoring and surveillance system in Georgia (GNMSS). Findings of this could provide policy makers with reliable, valid data for developing national strategy of micronutrient deficiency elimination and improving the nutritional status of population.

\section{Objectives of the study}

Objective1: Determining the prevalence of micronutrient deficiency (anemia, iron) in children.

Objective 2: Determining the prevalence of micronutrient deficiency (anemia, iron, and folate) in pregnant women.

\section{Study Design}

The study focused on following population groups including toddlers (aged 12 - 23 months old), and pregnant. Selection of these target groups were based on level of risk or vulnerability, accessibility of the target group for assessment, and degree of representativeness, or the ability to reflect the extent of the problem in the overall population. Other criteria included the availability of normative data and the potential usefulness of the targeted population for surveillance of other micronutrient deficiencies.

\section{Sites and Population}

With the support of USA CDC, in 2015, NCDC of Georgia launched collaborative project - "strengthening of micronutrient deficiency surveillance in Georgia". We have selected 8 sentinel sites ( 2 sites in each region/children and pregnant health facilities)in four regions of Georgia (Tbilisi, Kakheti, Achara, and Samegrelo), using the criteria of geographical, social, ethnical, urban/rural, and religion. We also identified existing information about malnutrition and dietary habits from the above mentioned regions.

The project protocol was approved by the Institutional review board (IRB) at the NCDC and by the Research Review Committee and Ethical review committee of the US CDC.

\section{Laboratory Methods}

1. After explaining the aim of the study (informal consent), a blood sample was collected from the antecubital vein in heparinized tube.

2. The sample for the test was taken from a vein and is placed in an appropriate test tube (a tube with granules and clot activator). Next, the sample is processed and the serum is separated from the clot.

3. Iron deficiency in survey participants has been measured using ferritin concentration in serum. Ferritin was tested in serum using the ELISA method. Hemoglobin was measured at the sentinel sites.

4. Folate deficiency has been measured using serum folate concentration measured on serum separated from blood. Testing was done using an ELISA method and a microbiologic test kit (DRG International Inc., USA. BIO-4886)

\section{Data Analysis}

The Statistic Package for the Social Sciences (SPSS) was used for Data Analysis.

\section{Results}

The study in pregnant women showed $7.4 \%$ prevalence of anemia in 2790 pregnant enrolling in study; the percentage of iron deficiency in total (483 pregnant with Lab. study on ferritin) resulted in $61 \%$ ). And $26.4 \%$ of folate deficiency in 483 pregnant (with Lab. study on folate).

The study in children (12-23 months of age) showed $34 \%$ prevalence of anemia in 939 children enrolling in study; the percentage of iron deficiency in total (478 children with Lab. study on ferritin) resulted in $84 \%)$.

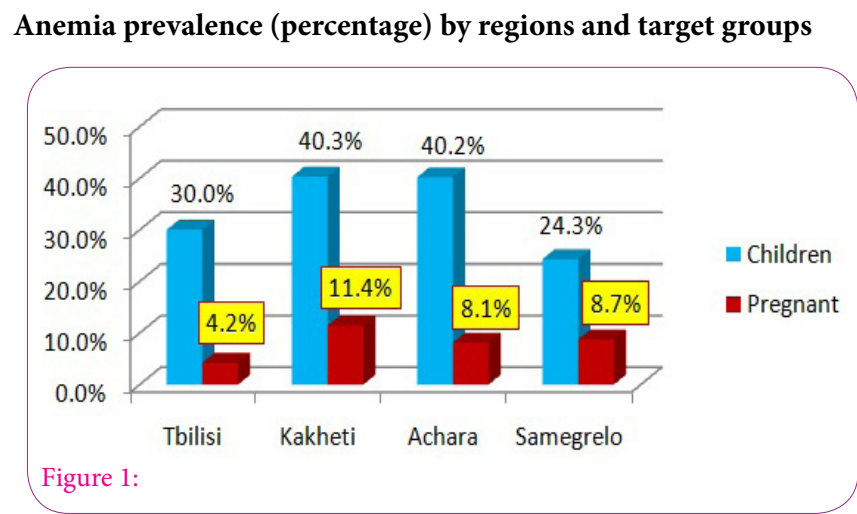

Severe anemia cases were not identified in both groups, not in toddlers and not in pregnant women.

In regards to regional profiles, we have detected that prevalence in toddlers in Samegrelo region (24.3\%) anemia percentage is 1.5 2 times less than in other three regions, especially when comparing with Achara region (40.2\%), which has the highest result compared to the other two regions (Kakheti with $40.3 \%$ and Tbilisi-30.0\%). Total percentage for all four regions is $34 \%$. (Table 1 ).

Reviewing regional profiles for anemia in pregnant, showed that anemia prevalence in studied 4 regions is almost the same, and the percentage is around the $10 \%$ (7.4\% in total), but we have a big differences in folate deficiency between Tbilisi and Achara regions (19.2\% in Tbilisi vs $37.2 \%$ in Achara).

\section{Discussion}

This study surveyed micronutrient deficiencies of three nutrition indicators (iron, folate, and iodine) in three target groups (children of 12-23 months and 12-12.99 years old children and pregnant women in the first trimester).

According to $\mathrm{WHO} / \mathrm{CDC}$ guidelines (ref), the prevalence of anemia in children population is classified as a "moderate" public health threat (by the public health significance of deficiency), but in pregnant, we have prevalence of "mild" deficiency. In terms of the folate deficiency, prevalence is high in pregnant women.

Comparing with other relevant studies, our data on anemia prevalence in children is 2.5 times higher than in Armenia but in child bearing women we have slightly lower prevalence.

Due to the project design, we could not define reasons of deficiency which require further investigation to determine proper intervention strategy. 


\begin{tabular}{|l|l|l|l|l|l|l|}
\hline Years & $\begin{array}{l}12-23 \text { moths age } \\
\text { children }\end{array}$ & $\begin{array}{l}\text { anemia } \mathrm{Hb}<110 \\
\mathrm{~g} / \mathrm{L}\end{array}$ & $\begin{array}{l}\text { Severe anemia } \mathrm{Hb} \\
<70 \mathrm{~g} / \mathrm{L}\end{array}$ & $\begin{array}{l}\text { pregnant }\left(1^{\text {st }}\right. \\
\text { trimester })\end{array}$ & $\begin{array}{l}\text { anemia } \mathrm{Hb}<110 \\
\mathrm{~g} / \mathrm{L}\end{array}$ & $\begin{array}{l}\text { anemia } \mathrm{Hb}<110 \\
\mathrm{~g} / \mathrm{L}\end{array}$ \\
\hline 2016 & 516 & $33.7 \%$ & $0.0 \%$ & 1680 & $6.3 \%$ & $0.0 \%$ \\
\hline 2017 & 423 & $34.3 \%$ & $0.0 \%$ & 1110 & $8.4 \%$ & $0.0 \%$ \\
\hline Total & 939 & $34 \%$ & $0.0 \%$ & 2790 & $7.4 \%$ & $0.0 \%$ \\
\hline
\end{tabular}

Due to similar studies in similar context, we can assume that above mentioned deficiencies are caused due to possible reasons: a) Poor nutrition of Georgian population. b) Georgian foods do not contain sufficient amount of micronutrients. c) "Formula" for toddlers' nutrition does not contain the needed micronutrients that should meet physiological requirements of children after breastfeeding.

\section{Limitations}

This study has one main limitation: We defined the prevalence of Iron Deficiency Anemia (IDA) and Folate Deficiency, but could not investigate the reasons of the deficiency because, the study project did not include personal interviews on dietary habits.

\section{Conclusion}

After reviewing of preliminary analyses of the sentinel approach surveillance system's 2 years (2016-2017 yy) functioning results we can conclude that: we have anemia problem in children U2; Folate deficiency problem in pregnant; Iron deficiency problem, that is reflection of the general situation existing in Georgia. According to experts opinion it is the quite sufficient evidences for final conclusion and recommendations for the initiation of nutrition interventions (mainly, food fortification) and for making the adequate changes/ amendments in relative legislation. Exception of provision of Children (6-23 months) from social unprotected families with Multi micronutrients powder.

\section{Recommendations}

1. Need to advocate for nutrition interventions regarding food fortification (with iron and folic acid) strategy.

2. Should implement one additional project for studying the dietary habits of population using standard questionnaires.

3. Promote the main principle of healthy eating.

4. In toddlers, special nutrition powder can be used to supplement feeding menus.

\section{Funding}

This research study was funded by the United States Centers for Disease Control and Prevention (Strengthening micronutrients deficiency surveillance in Georgia- grant \# 5U2GGH001658).

\section{Acknowledgements}

The research team/NCDC acknowledges with gratitude the commitment of the Centers for Disease Control and Prevention to the research efforts.

Staff of sentinel sites (Health Facilities) and Laboratories involved in the project.

Thank to Dorothy L. Southern for providing scientific writing advice and critically reviewing the manuscript.

\section{References}

1. The Epidemiology of Global Micronutrient Deficiencies/Bailey.

2. Armenia Demographic and Health Survey.

3. GNS -2009.

4. Iron Deficiency Anaemia Assessment, Prevention and Control. A guide for programme managers.

5. Conclusions of a WHO Technical Consultation on folate and vitamin B12 deficiencies. Food and Nutrition Bulletin. The United Nations University. 